\title{
Highly Diastereoselective Vinylogous Mukaiyama Aldol Reaction of Isatins with 2-(Trimethylsilyloxy)furans Catalyzed by Quinine
}

Shasha Kong, ${ }^{\mathrm{a}}$ Weidong Fan, ${ }^{\mathrm{a}}$ Hairong Lyu, ${ }^{\mathrm{a}}$ Junchen Zhan, ${ }^{\mathrm{a}}$ Xinyu Miao, ${ }^{\mathrm{b}}$ and Zhiwei Miao*a

${ }^{a}$ State Key Laboratory of Elemento-Organic Chemistry, Research Institute of Elemento-Organic Chemistry, Nankai University, Tianjin, China. E-mail: miaozhiwei@nankai.edu.cn; Fax: +86 222350 2351; Tel: +86 2223502351

${ }^{b}$ Tianjin Yaohua High School, Tianjin, 300040, P. R. China 


\section{Supporting Information}

\section{Experimental Section}

\section{General Comments.}

All reactions were carried out under an inert atmosphere and in heat-dried glassware. Anhydrous THF were obtained by distillation from sodium. Flash column chromatography was performed on silica gel (particle size 10-40 $\mu \mathrm{m}$, Ocean Chemical Factory of Qingdao, China). Nitrogen gas (99.999\%) was purchased from Boc Gas Inc. ${ }^{1} \mathrm{H}$ NMR and ${ }^{13} \mathrm{C}$ NMR spectra were recorded on

Bruker-400 (400 MHz for ${ }^{1} \mathrm{H}, 101 \mathrm{MHz}$ for $\left.{ }^{13} \mathrm{C}\right)$ spectrometers. Chemical shifts were reported in ppm downfield from internal $\mathrm{Si}\left(\mathrm{CH}_{3}\right)_{4}$. The crystal structure was determined on a Bruker SMART 1000 CCD diffractometer. Mass spectra were recorded on a LCQ advantage spectrometer with ESI resource. HR-MS were recorded on APEXII and ZAB-HS spectrometer. Melting points were determined on a T-4 melting point apparatus (uncorrected).

\section{Typical procedure for quinine catalyzed vinylogous Mukaiyama aldol reaction:}

To a solution of isatin $1(0.5 \mathrm{mmol})$ in THF $(2 \mathrm{~mL})$ was added quinine $(0.017 \mathrm{~g}, 10 \mathrm{~mol} \%)$. The mixture was cooled to $-78{ }^{\circ} \mathrm{C}$ and stirred at this temperature for 15 minutes. Then the solution of 2-(trimethylsilyloxy)furan $2(0.156 \mathrm{~g}, 1 \mathrm{mmol})$ in THF $(1 \mathrm{~mL})$ was added dropwise. The mixture was stirred at $-78^{\circ} \mathrm{C}$ for 15 minutes. The reaction was monitored by TLC. After the completion of the reaction it was quenched by addition of $10 \%$ aqueous $\mathrm{HCl}(2.0 \mathrm{~mL})$ and the mixture was extracted with ethyl acetate $(3 \times 5 \mathrm{~mL})$. The combined organic layer was washed with brine solution, dried over $\mathrm{MgSO}_{4}$, and concentrated under reduced pressure (rotary evaporator). The crude products were purified by silica gel column chromatography (AcOEt/hexane) to afford the product as a white solid. 
3-(2,5-Dihydro-5-oxofuran-2-yl)-3-hydroxyindolin-2-one (3a).<smiles>O=C1C=CC(C2(O)C(=O)Nc3ccccc32)O1</smiles>

Yield: $103 \mathrm{mg}$, (89\%); white solid; mp 190-192 ${ }^{\circ} \mathrm{C} .{ }^{1} \mathrm{H}$ NMR (400 MHz, DMSO): $\delta 10.44(\mathrm{~s}, 1 \mathrm{H}), 7.73(\mathrm{~d}, J=5.6 \mathrm{~Hz}, 1 \mathrm{H}), 7.32(\mathrm{~d}, J=7.3 \mathrm{~Hz}, 1 \mathrm{H})$, $7.25(\mathrm{t}, J=7.6 \mathrm{~Hz}, 1 \mathrm{H}), 6.99(\mathrm{t}, J=7.5 \mathrm{~Hz}, 1 \mathrm{H}), 6.82(\mathrm{~d}, J=7.7 \mathrm{~Hz}, 1 \mathrm{H})$, $6.77(\mathrm{~s}, 1 \mathrm{H}), 6.21(\mathrm{~d}, J=4.7 \mathrm{~Hz}, 1 \mathrm{H}), 5.45(\mathrm{~s}, 1 \mathrm{H}) .{ }^{13} \mathrm{C}$ NMR (101 MHz, DMSO): $\delta$ 175.09, 172.50, $154.42,141.92,129.88,128.24,124.62,122.08,121.81,109.89,85.09,76.65$. HRMS (ESI): $\mathrm{m} / \mathrm{z}$ calcd for $\left[\mathrm{C}_{12} \mathrm{H}_{9} \mathrm{NO}_{4}+\mathrm{Na}\right]^{+}: 254.0424[\mathrm{M}+\mathrm{Na}]^{+}$; found 254.0421 .

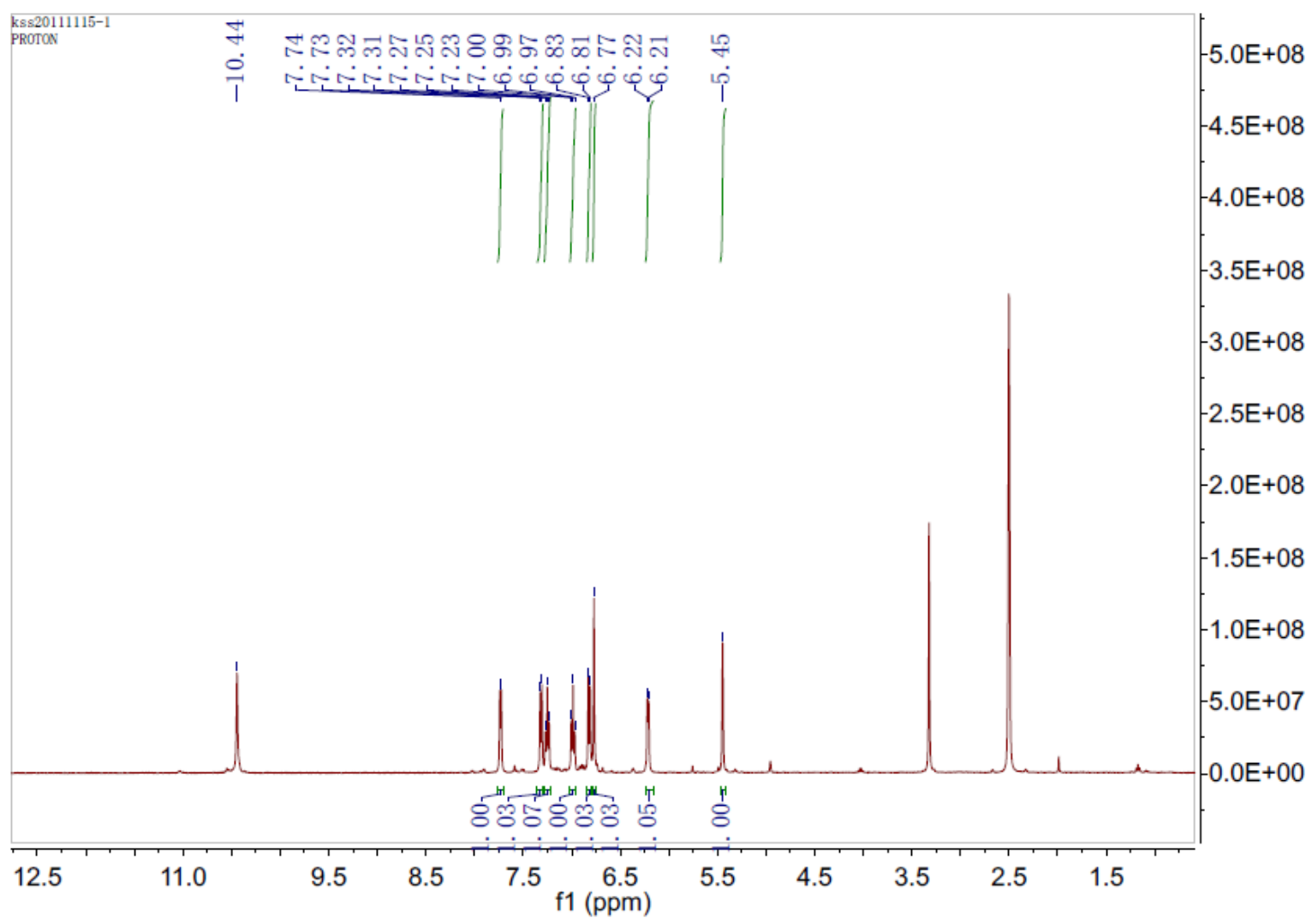




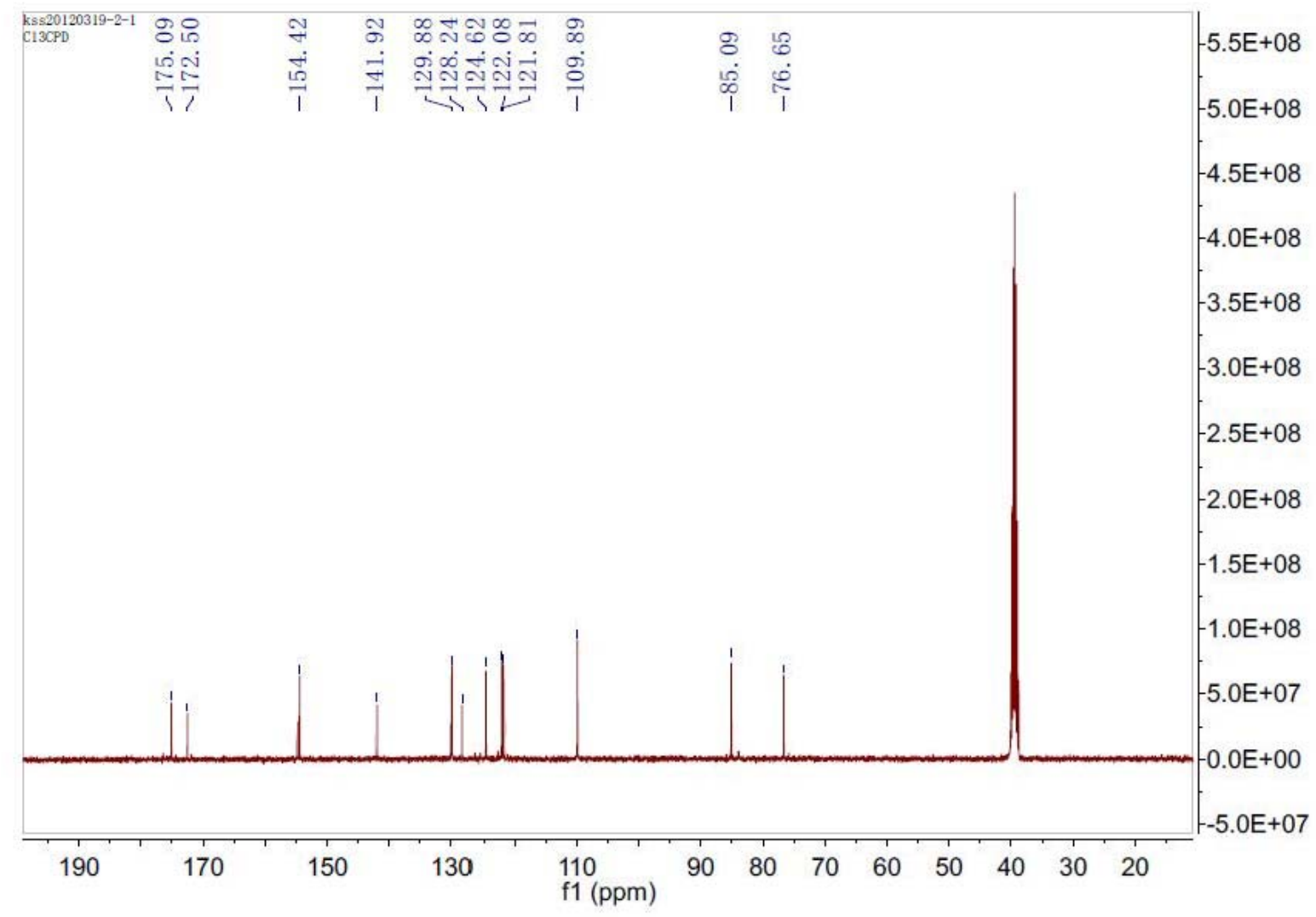

3-(2,5-Dihydro-5-oxofuran-2-yl)-3-hydroxy-5-methylindolin-2-one (3b).<smiles>Cc1ccc2c(c1)C(O)([C@H]1C=CC(=O)O1)C(=O)N2</smiles>

Yield: $122 \mathrm{mg}$, (89\%); white solid; mp 201-204 ${ }^{\circ} \mathrm{C} .{ }^{1} \mathrm{H}$ NMR (400 MHz, DMSO): $\delta 10.44(\mathrm{~s}, 1 \mathrm{H}), 8.01(\mathrm{~d}, J=5.8 \mathrm{~Hz}, 1 \mathrm{H}), 7.05(\mathrm{~d}, J=7.8 \mathrm{~Hz}$, $1 \mathrm{H}), 6.87(\mathrm{~s}, 1 \mathrm{H}), 6.70-6.71(\mathrm{~m}, 1 \mathrm{H}), 6.69(\mathrm{~s}, 1 \mathrm{H}), 6.38(\mathrm{dd}, J=5.7,1.7$ $\mathrm{Hz}, 1 \mathrm{H}), 5.30$ (s, 1H), 2.21 (s, 3H). ${ }^{13} \mathrm{C}$ NMR (101 MHz, DMSO): $\delta$ 176.33, 171.94, 154.74, 140.00, 130.19, 129.98, 126.21, 125.26, 122.62, 109.60, 84.02, 75.87, 20.62. HRMS (ESI): $\mathrm{m} / \mathrm{z}$ calcd for $\left[\mathrm{C}_{13} \mathrm{H}_{11} \mathrm{NO}_{4}+\mathrm{Na}\right]^{+}: 268.0580[\mathrm{M}+\mathrm{Na}]^{+}$; found 268.0585. 

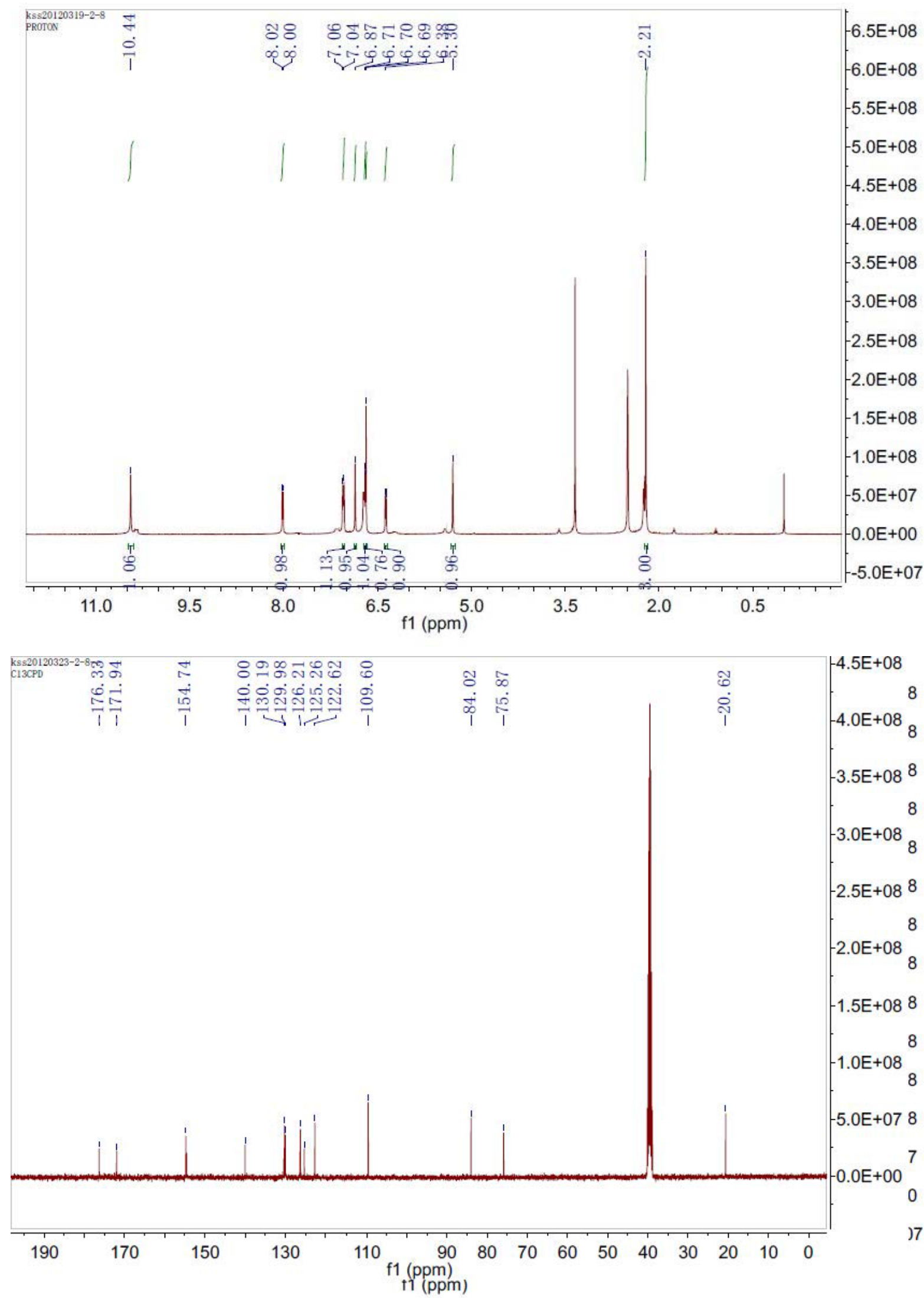
7-Chloro-3-(2,5-dihydro-5-oxofuran-2-yl)-3-hydroxyindolin-2-one (3c).

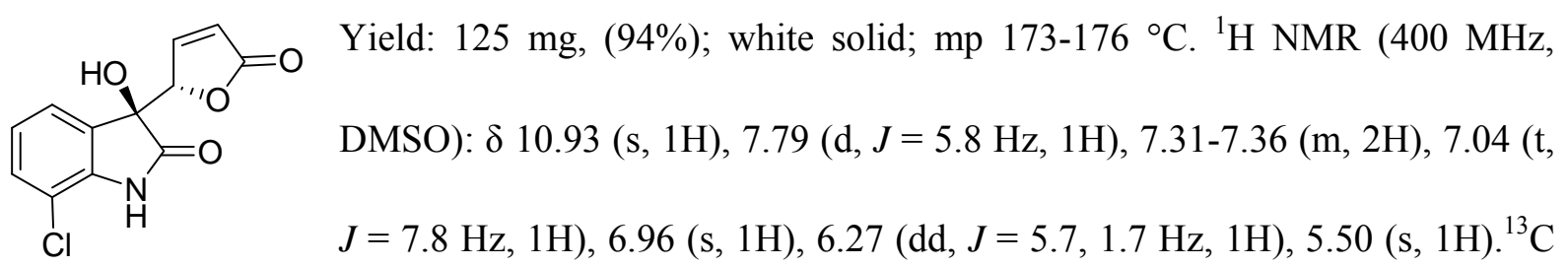

NMR (101 MHz, DMSO): $\delta$ 174.97, 172.31, 154.38, 139.67, 130.44, 129.86, 124.16, 123.25,

122.14, 114.10, 84.74, 77.15. HRMS (ESI): $\mathrm{m} / \mathrm{z}$ calcd for $\left[\mathrm{C}_{12} \mathrm{H}_{8} \mathrm{ClNO}_{4}+\mathrm{Na}\right]^{+}: 288.0039[\mathrm{M}+\mathrm{Na}]^{+}$; found 288.0043 .

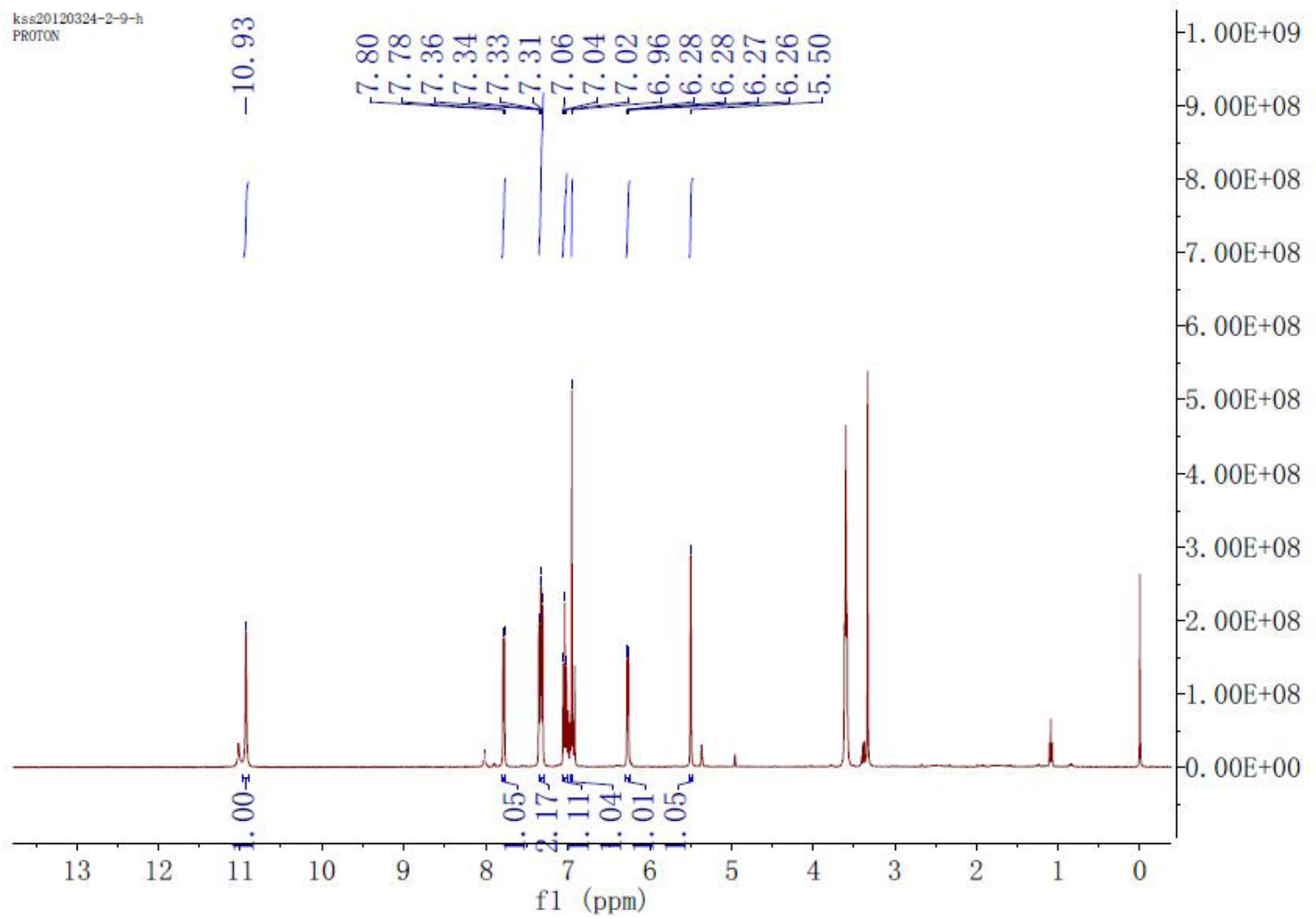


3-(2,5-Dihydro-5-oxofuran-2-yl)-3-hydroxy-5-methoxyindolin-2-one (3d).<smiles>COc1ccc2c(c1)[C@](O)([C@H]1C=CC(=O)O1)C(=O)N2</smiles>

Yield: $116 \mathrm{mg}$, (89\%); white solid; mp 204-207 ${ }^{\circ} \mathrm{C} .{ }^{1} \mathrm{H}$ NMR (400 MHz, DMSO): $\delta 10.40$ (s, 1H), $8.04(\mathrm{~s}, 1 \mathrm{H}), 6.84-6.98(\mathrm{~m}, 2 \mathrm{H}), 6.67$ (s, 1H), $6.41(\mathrm{~s}, 1 \mathrm{H}), 5.35(\mathrm{~s}, 1 \mathrm{H}), 3.70(\mathrm{~s}, 3 \mathrm{H}), 3.38(\mathrm{~s}, 1 \mathrm{H}) .{ }^{13} \mathrm{C} \mathrm{NMR}$ (101 MHz, DMSO): $\delta 176.22,171.89,154.80,135.61,127.70,122.75,114.31,112.84,111.53$ 110.21, 83.98, 76.16, 55.44. HRMS (ESI): $\mathrm{m} / \mathrm{z}$ calcd for $\left[\mathrm{C}_{13} \mathrm{H}_{11} \mathrm{NO}_{5}+\mathrm{Na}\right]^{+}: 284.0529[\mathrm{M}+\mathrm{Na}]^{+}$; found 284.0532 .

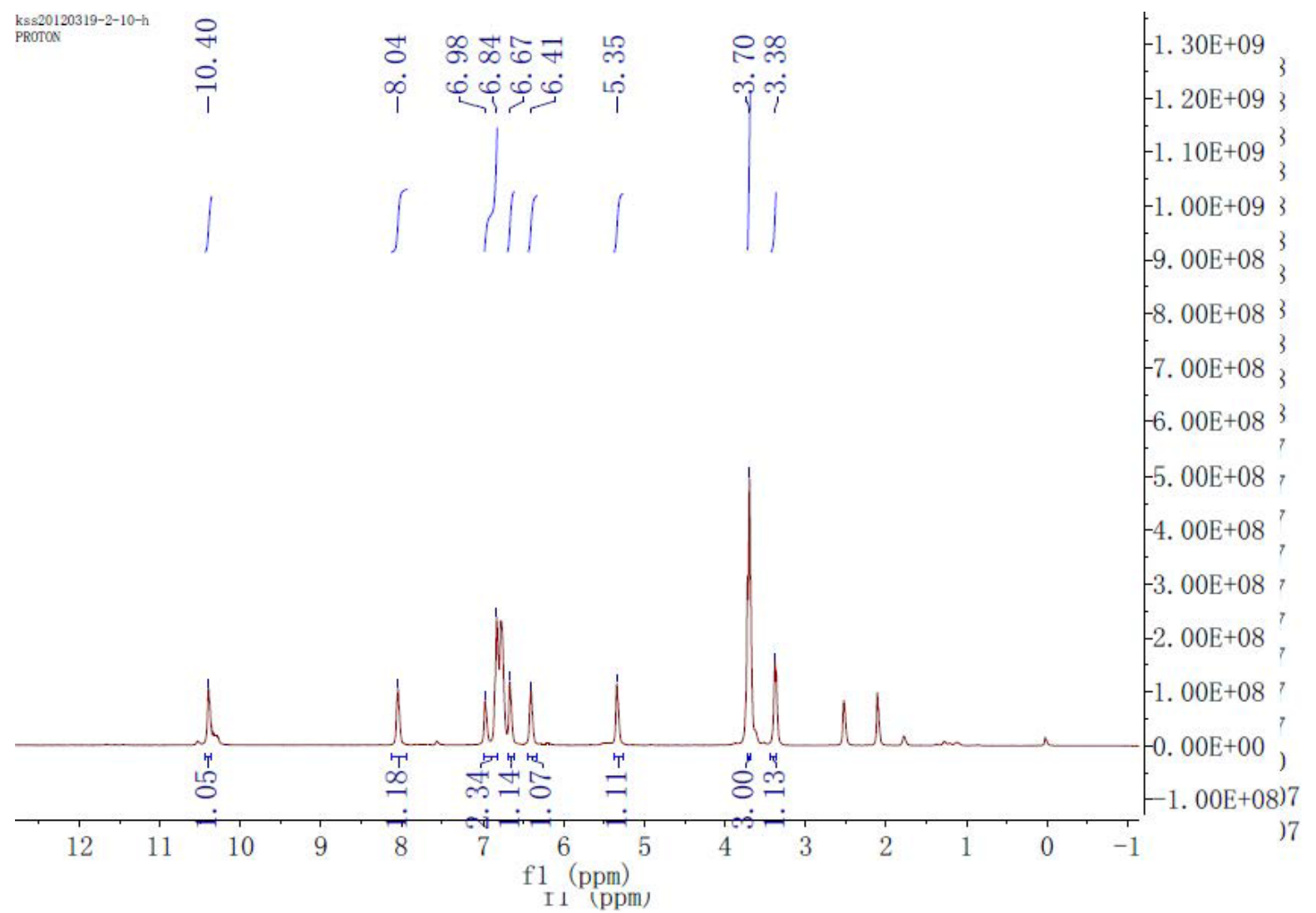




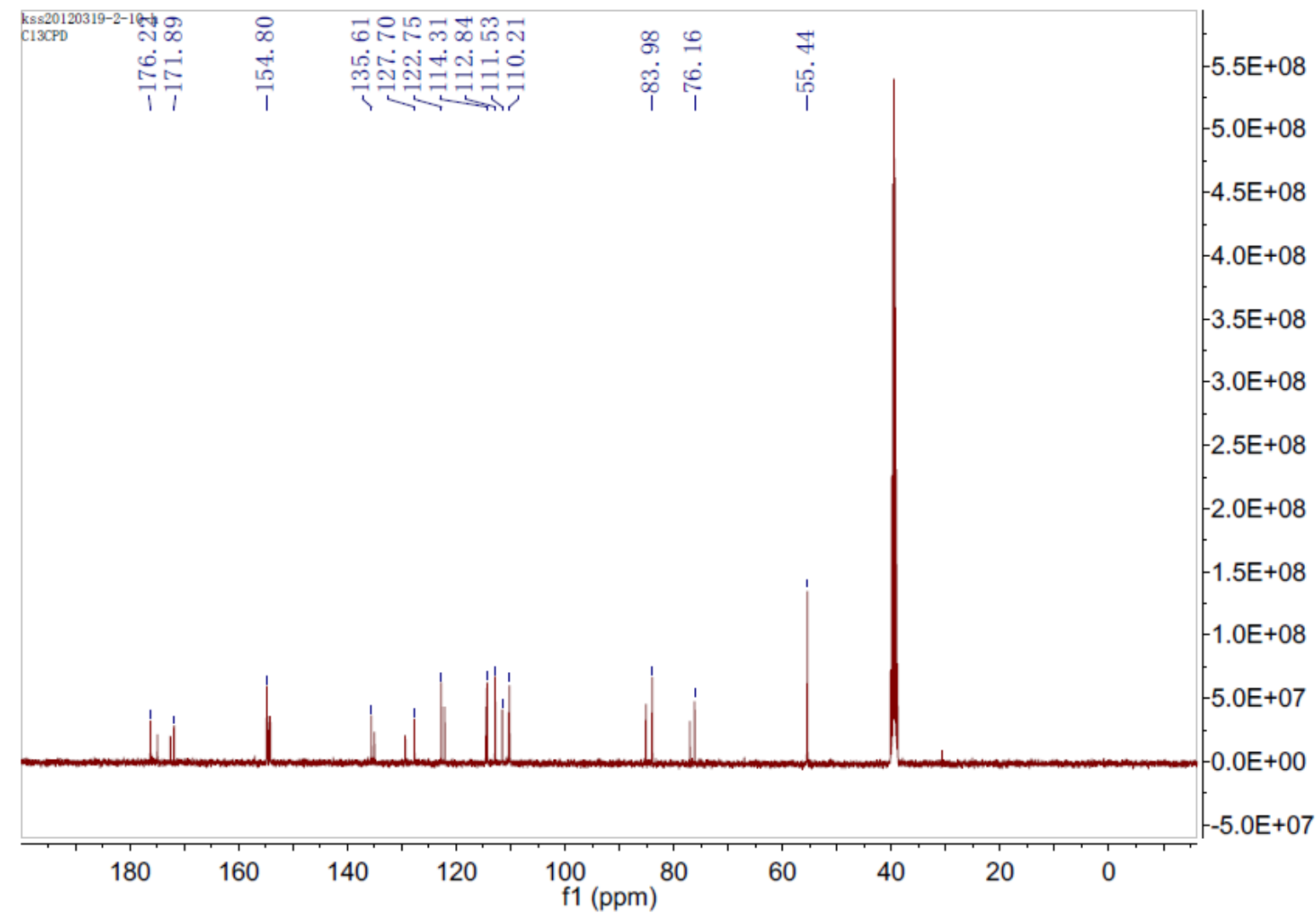

5-Bromo-3-(2,5-dihydro-5-oxofuran-2-yl)-3-hydroxyindolin-2-one (3e).

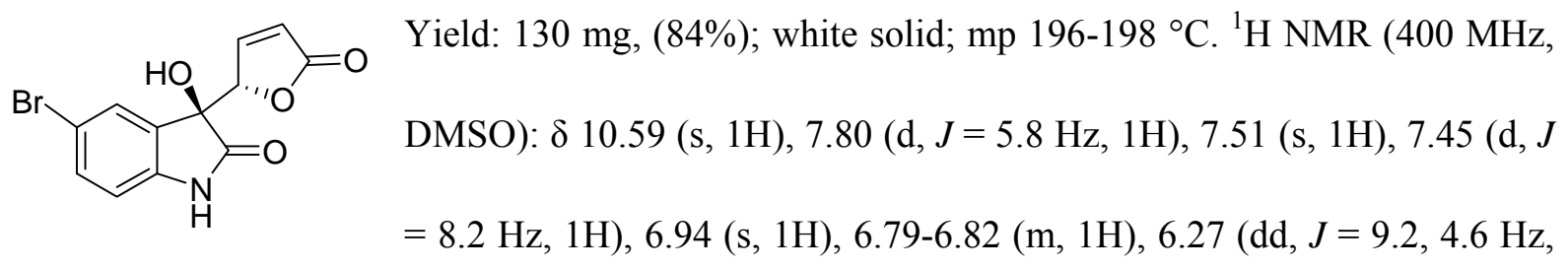

1H), 5.52 (s, 1H). ${ }^{13} \mathrm{C}$ NMR (101 MHz, DMSO): $\delta 174.50,172.30,154.61,141.30,132.56,130.88$, 127.52, 122.04, 113.47, 111.93, 84.66, 76.68. HRMS (ESI): $\mathrm{m} / \mathrm{z}$ calcd for $\left[\mathrm{C}_{12} \mathrm{H}_{8} \mathrm{BrNO}_{4}+\mathrm{Na}\right]^{+}$: $331.9529[\mathrm{M}+\mathrm{Na}]^{+}$; found 331.9525 . 


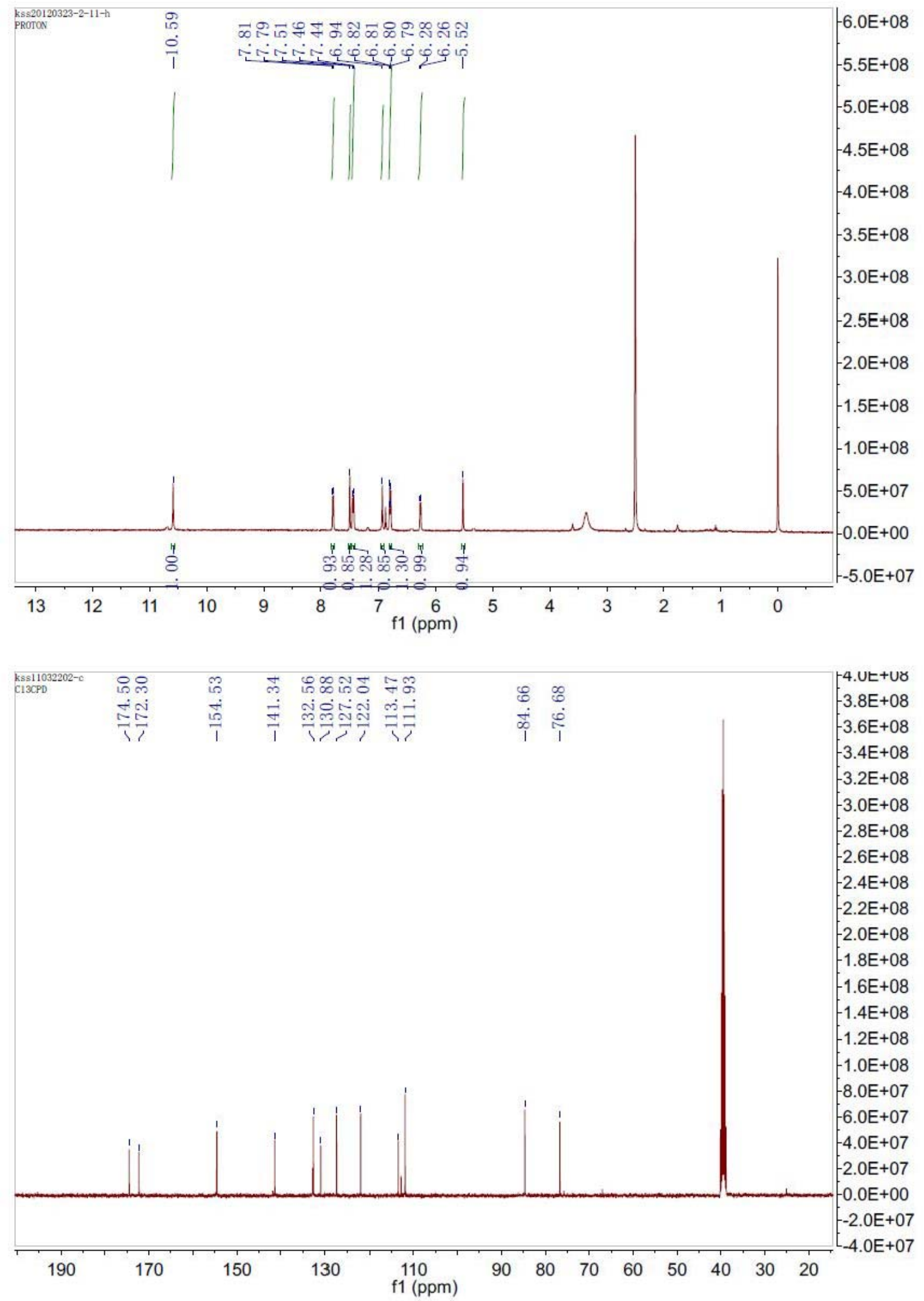


3-(2,5-Dihydro-5-oxofuran-2-yl)-3-hydroxy-5,7-dimethylindolin-2-one (3f).<smiles>Cc1cc(C)c2c(c1)[C@](O)([C@H]1C=CC(=O)O1)C(=O)N2</smiles>

Yield: 112 mg, (87\%); white solid; mp 190-192 ${ }^{\circ} \mathrm{C} .{ }^{1} \mathrm{H}$ NMR (400 MHz, DMSO): $\delta 10.41(\mathrm{~s}, 1 \mathrm{H}), 7.75(\mathrm{~d}, J=5.8 \mathrm{~Hz}, 1 \mathrm{H}), 6.98(\mathrm{~s}, 1 \mathrm{H}), 6.90(\mathrm{~s}$, $1 \mathrm{H}), 6.69(\mathrm{~s}, 1 \mathrm{H}), 6.24(\mathrm{dd}, J=5.8,2.0 \mathrm{~Hz}, 1 \mathrm{H}), 5.42(\mathrm{~s}, 1 \mathrm{H}), 2.21(\mathrm{~s}$,

3H), 2.15 (s, 3H). ${ }^{13} \mathrm{C}$ NMR (101 MHz, DMSO): $\delta$ 175.50, 172.52, 154.66, 138.02, 131.45, 130.56, 128.19, 122.54, 121.91, 118.82, 85.12, 76.83, 20.56, 16.12. HRMS (ESI): $\mathrm{m} / \mathrm{z}$ calcd for $\left[\mathrm{C}_{14} \mathrm{H}_{13} \mathrm{NO}_{4}+\mathrm{Na}\right]^{+}: 282.0737[\mathrm{M}+\mathrm{Na}]^{+}$; found 282.0742.

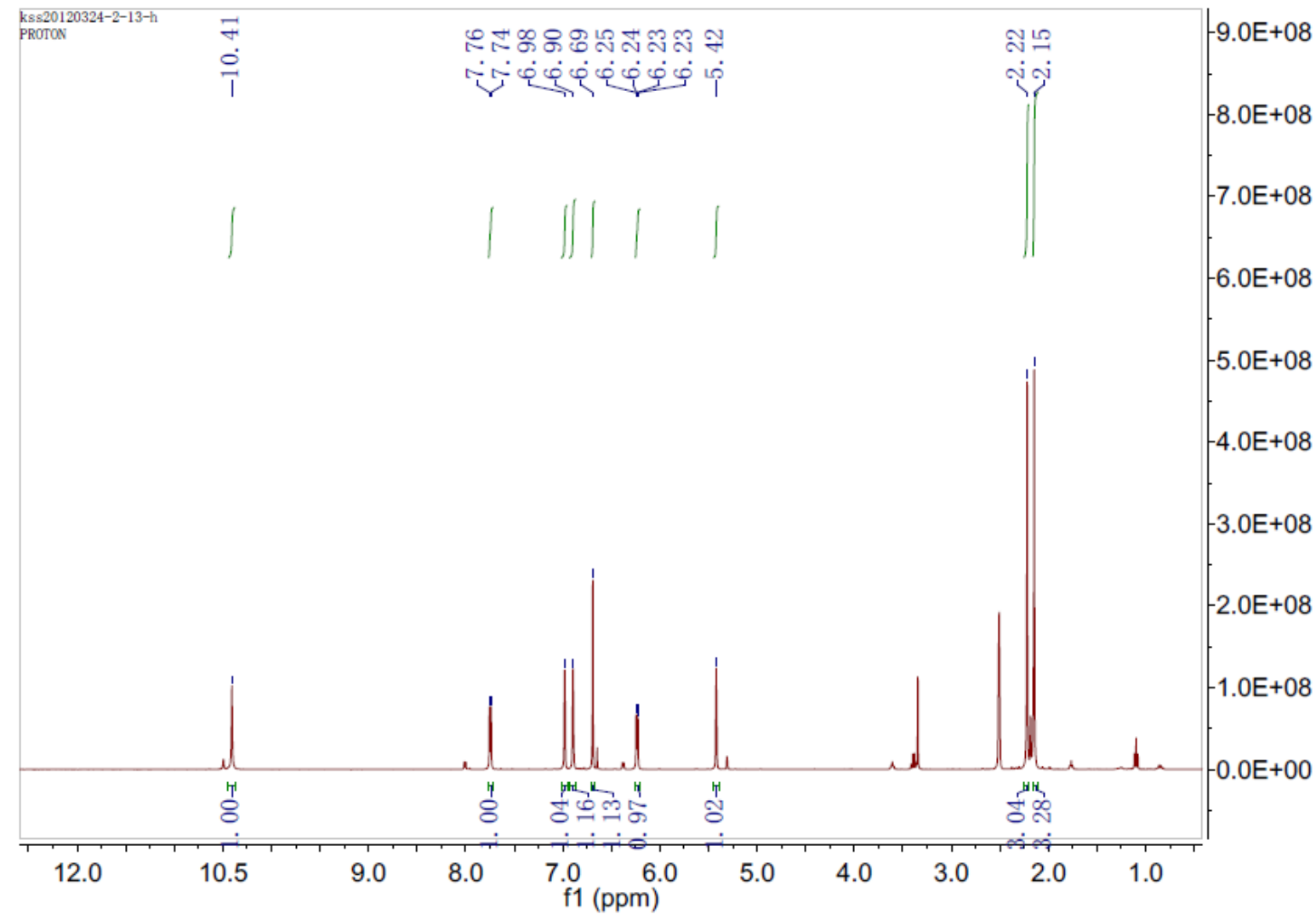




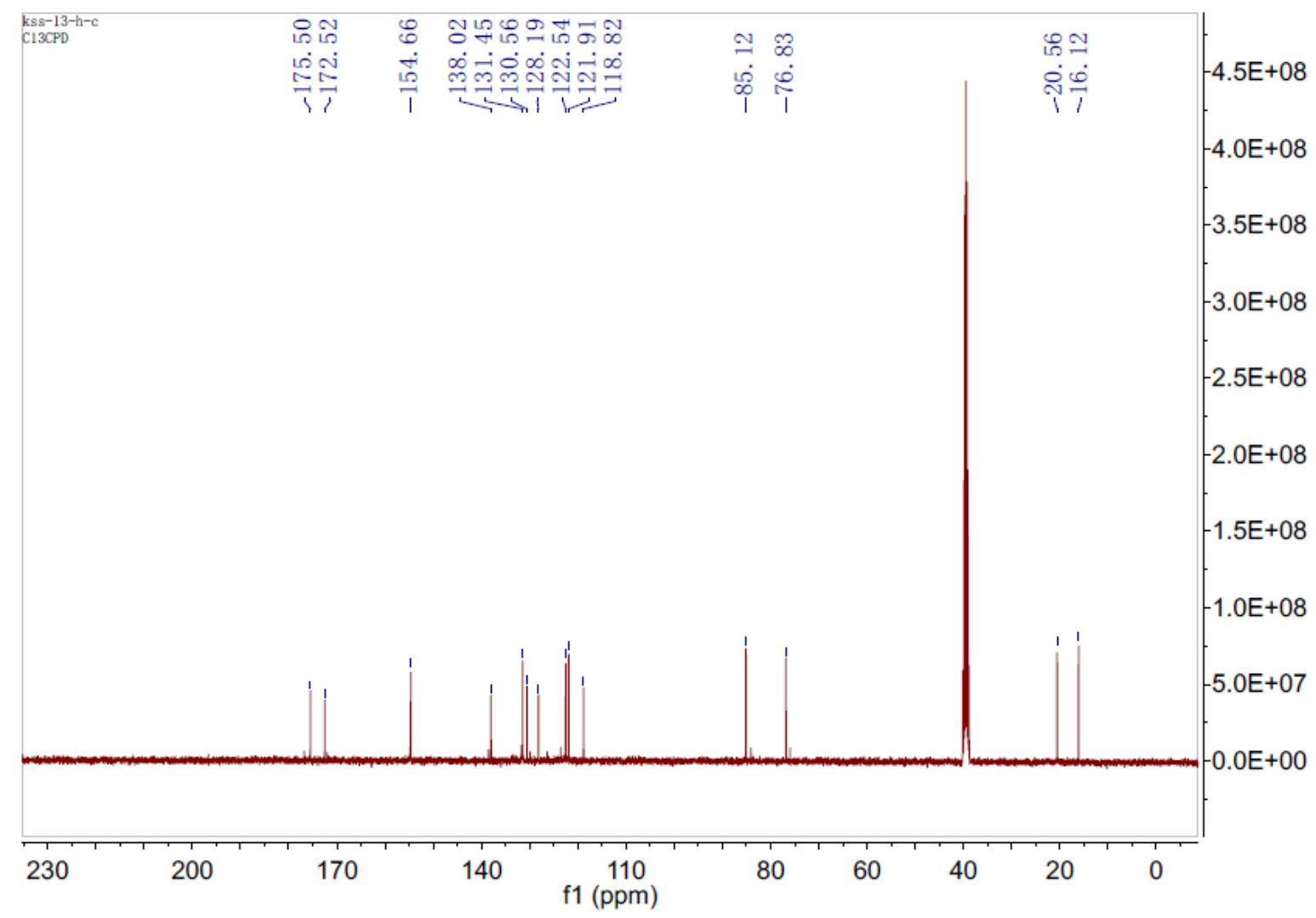

1-Benzyl-3-(2,5-dihydro-5-oxofuran-2-yl)-3-hydroxyindolin-2-one (3g).

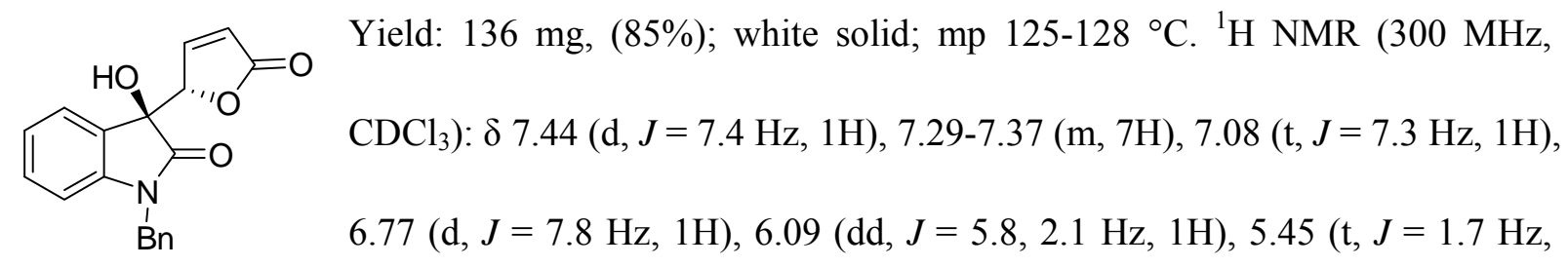

1H), $5.00(\mathrm{~d}, J=15.6 \mathrm{~Hz}, 1 \mathrm{H}), 4.76(\mathrm{~d}, J=15.6 \mathrm{~Hz}, 1 \mathrm{H}), 3.27(\mathrm{~s}, 1 \mathrm{H}) .{ }^{13} \mathrm{C} \mathrm{NMR}\left(101 \mathrm{MHz}, \mathrm{CDCl}_{3}\right)$ $\delta 174.06,172.05,151.43,142.44,134.80,130.69,129.00,127.98,127.20,126.15,125.54,124.71$, 123.79, 123.72, 109.97, 85.37, 44.02. HRMS (ESI): $\mathrm{m} / \mathrm{z}$ calcd for $\left[\mathrm{C}_{19} \mathrm{H}_{15} \mathrm{NO}_{4}+\mathrm{Na}\right]^{+}: 344.0893$ $[\mathrm{M}+\mathrm{Na}]^{+}$; found 344.0889 . 

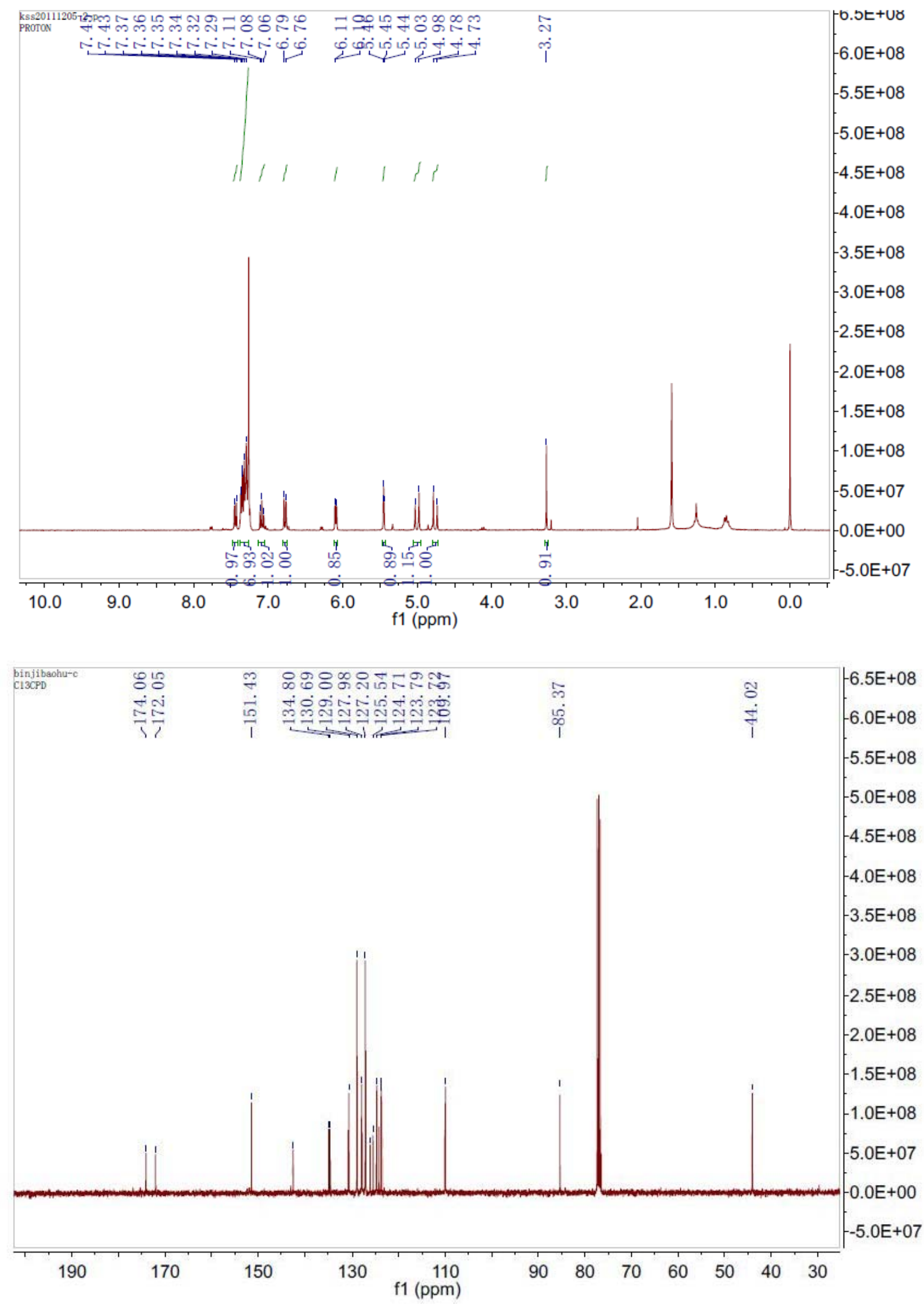
7-Chloro-3-(2,5-dihydro-5-oxofuran-2-yl)-3-hydroxy-1-methylindolin-2-one (3h).

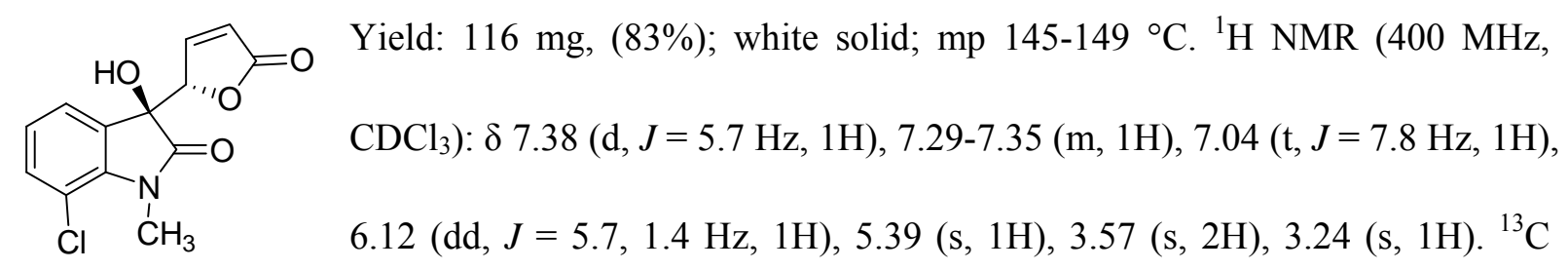

NMR $\left(101 \mathrm{MHz}, \mathrm{CDCl}_{3}\right): \delta 174.15,171.86,150.94,139.18,133.17,128.61,124.64,124.03$,

123.25, 116.51, 89.19, 84.11, 29.99. HRMS (ESI): $\mathrm{m} / \mathrm{z}$ calcd for $\left[\mathrm{C}_{13} \mathrm{H}_{10} \mathrm{NO}_{4} \mathrm{Cl}+\mathrm{Na}\right]^{+}: 302.0191$

$[\mathrm{M}+\mathrm{Na}]^{+}$; found 302.0189 .

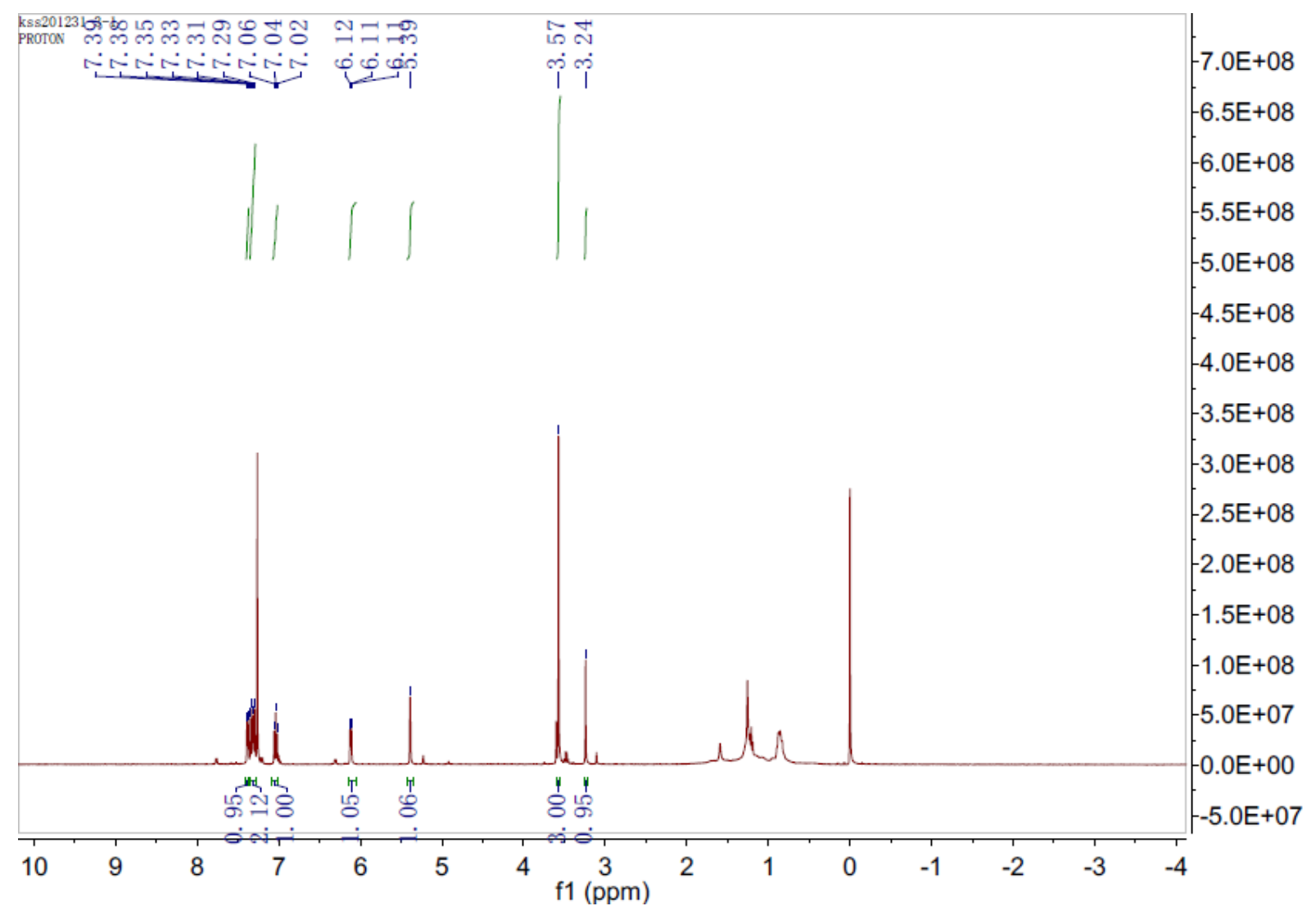




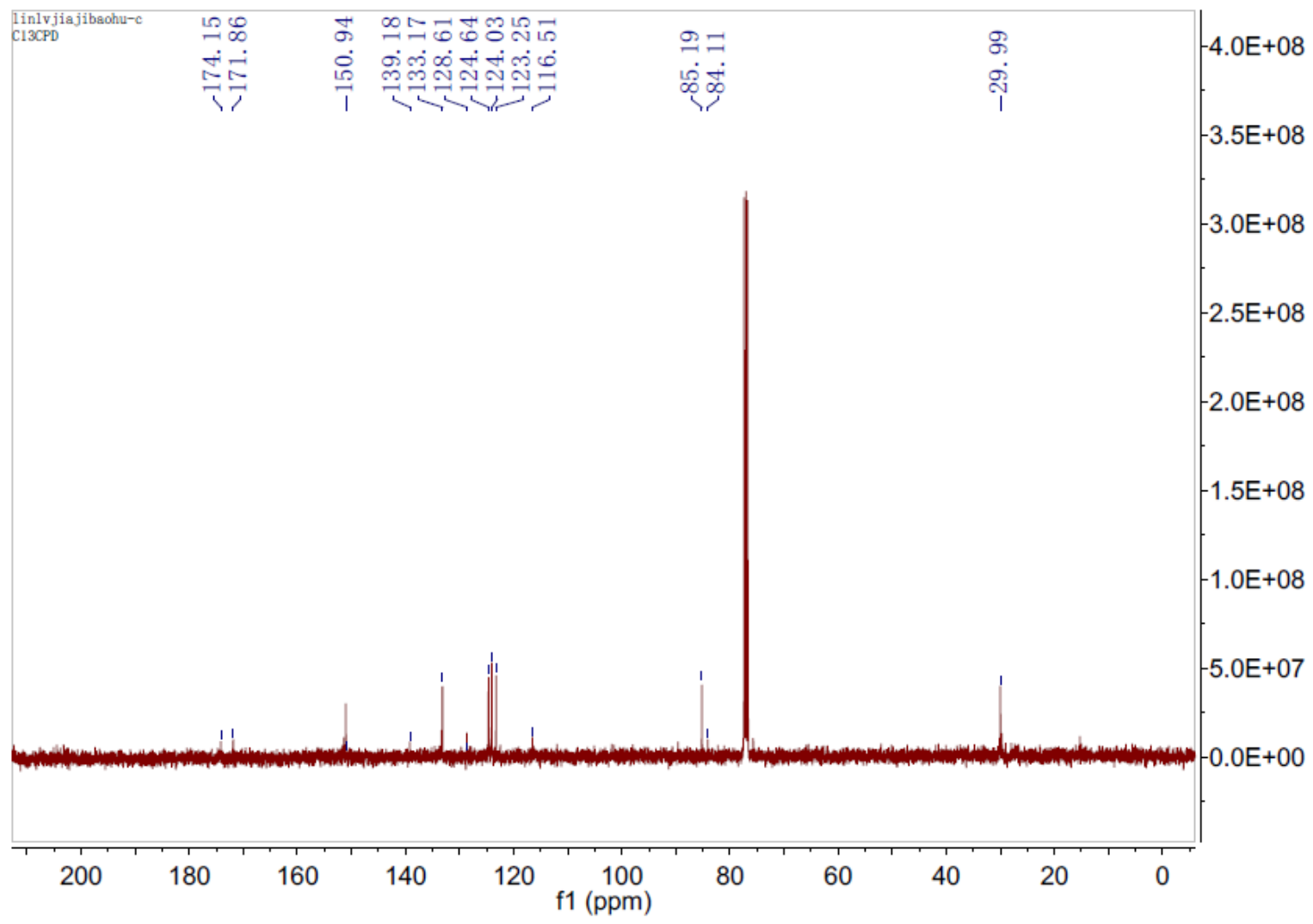

3-(2,5-Dihydro-5-oxofuran-2-yl)-3-hydroxy-1-methylindolin-2-one (3i).

Yield: $105 \mathrm{mg},(86 \%)$; white solid; mp 138-140 ${ }^{\circ} \mathrm{C} .{ }^{1} \mathrm{H}$ NMR $(400 \mathrm{MHz}$,
$\left.\mathrm{CDCl}_{3}\right): \delta 7.80(\mathrm{~d}, J=5.6 \mathrm{~Hz}, 1 \mathrm{H}), 7.38(\mathrm{t}, J=7.7 \mathrm{~Hz}, 2 \mathrm{H}), 7.07(\mathrm{t}, J=7.6 \mathrm{~Hz}$,

$\mathrm{Hz}, 1 \mathrm{H}), 3.55(\mathrm{~s}, 1 \mathrm{H}), 3.22(\mathrm{~d}, J=7.8 \mathrm{~Hz}, 3 \mathrm{H}) .{ }^{13} \mathrm{C} \mathrm{NMR}\left(101 \mathrm{MHz}, \mathrm{CDCl}_{3}\right): \delta 175.04,171.81$, 151.96, 143.82, 130.96, 125.49, 124.74, 124.19, 123.81, 123.19, 109.02, 84.20, 26.59. HRMS (ESI): $\mathrm{m} / \mathrm{z}$ calcd for $\left[\mathrm{C}_{13} \mathrm{H}_{11} \mathrm{NO}_{4}+\mathrm{Na}\right]^{+}: 268.0580[\mathrm{M}+\mathrm{Na}]^{+}$; found 268.0585 . 


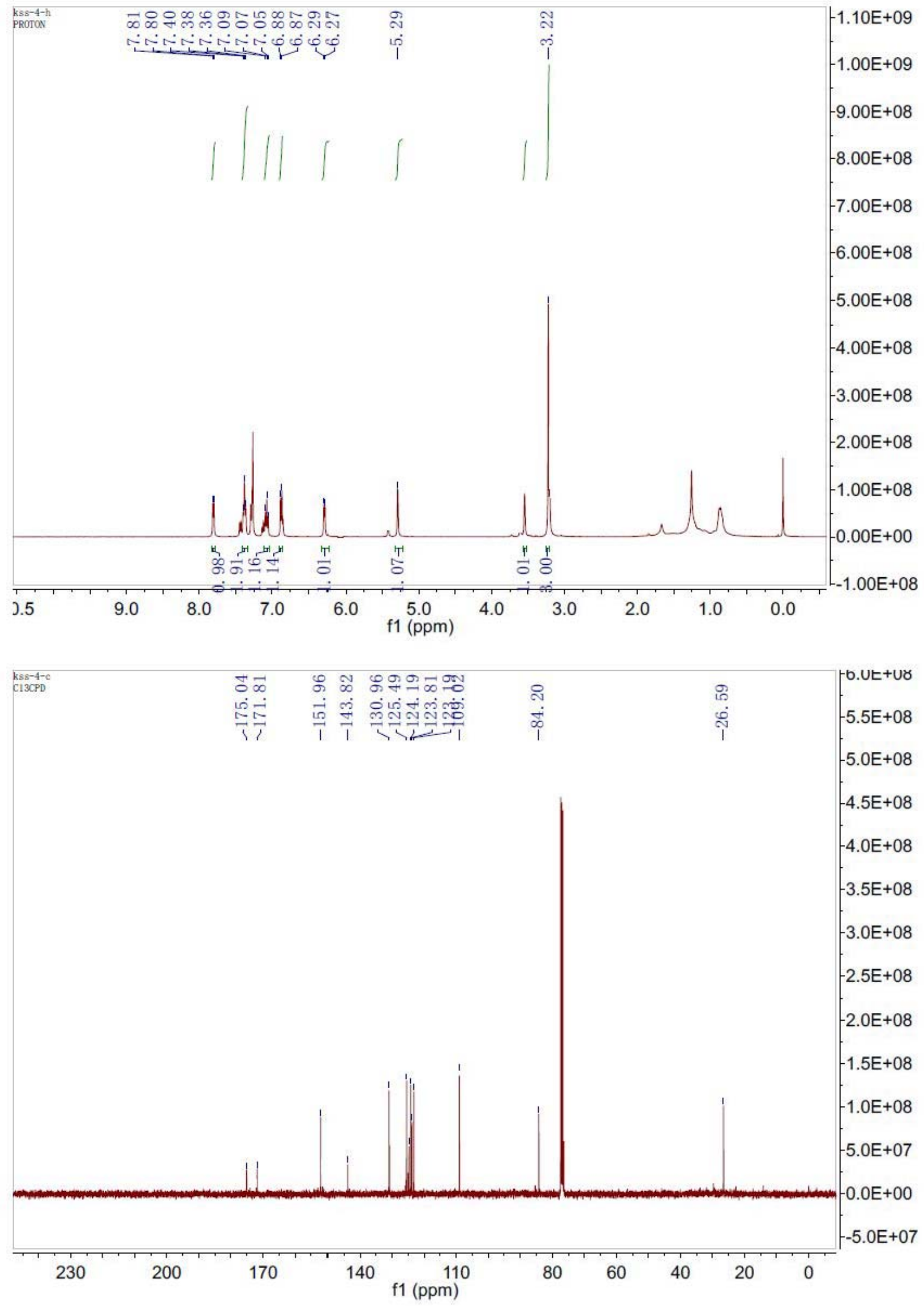


5-Chloro-3-(2,5-dihydro-5-oxofuran-2-yl)-3-hydroxy-1-methylindolin-2-one (3j).<smiles>CN1C(=O)C(O)(C2C=CC(=O)O2)c2cc(Cl)ccc21</smiles>

Yield: $118 \mathrm{mg},(85 \%)$; white solid; mp 156-159 ${ }^{\circ} \mathrm{C} .{ }^{1} \mathrm{H}$ NMR $(400 \mathrm{MHz}$, $\left.\mathrm{CDCl}_{3}\right) \delta: 7.82(\mathrm{dd}, J=5.8,1.3 \mathrm{~Hz}, 1 \mathrm{H}), 7.35(\mathrm{dd}, J=8.3,2.0 \mathrm{~Hz}, 1 \mathrm{H})$, $7.27(\mathrm{~d}, J=2.0 \mathrm{~Hz}, 1 \mathrm{H}), 6.80(\mathrm{~d}, J=8.3 \mathrm{~Hz}, 1 \mathrm{H}), 6.32(\mathrm{dd}, J=5.7,1.8$ $\mathrm{Hz}, 1 \mathrm{H}), 5.27(\mathrm{~s}, 1 \mathrm{H}), 3.97(\mathrm{~s}, 1 \mathrm{H}), 3.21(\mathrm{~s}, 3 \mathrm{H}) .{ }^{13} \mathrm{C} \mathrm{NMR}\left(101 \mathrm{MHz}, \mathrm{CDCl}_{3}\right): \delta 174.61,171.65$ $151.59,142.34,130.86,128.72,126.70,126.05,125.16,124.48,110.01,83.94,26.76$. HRMS (ESI): $\mathrm{m} / \mathrm{z}$ calcd for $\left[\mathrm{C}_{13} \mathrm{H}_{10} \mathrm{NO}_{4} \mathrm{Cl}+\mathrm{Na}\right]^{+}: 302.0191[\mathrm{M}+\mathrm{Na}]^{+}$; found 302.0190.

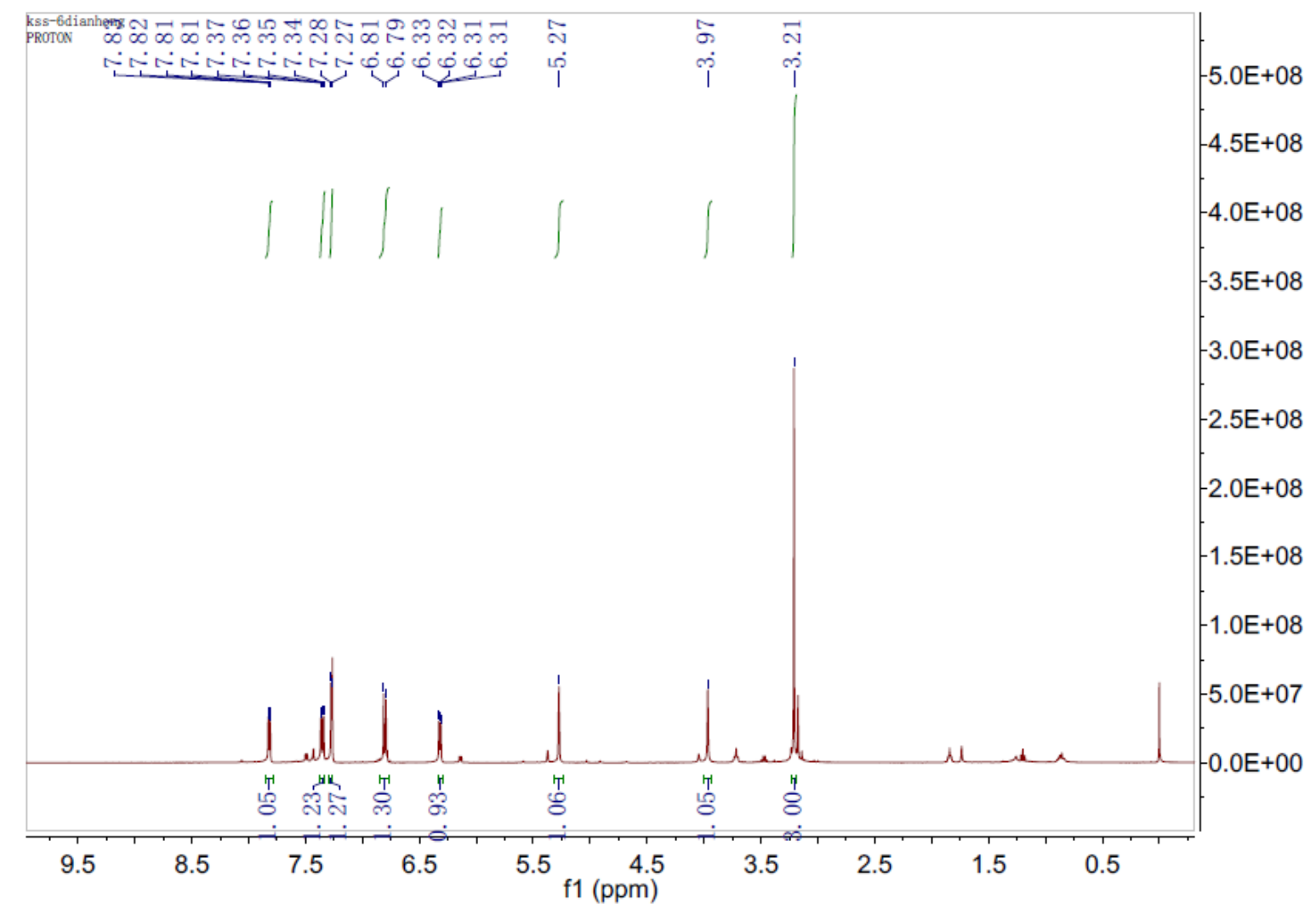




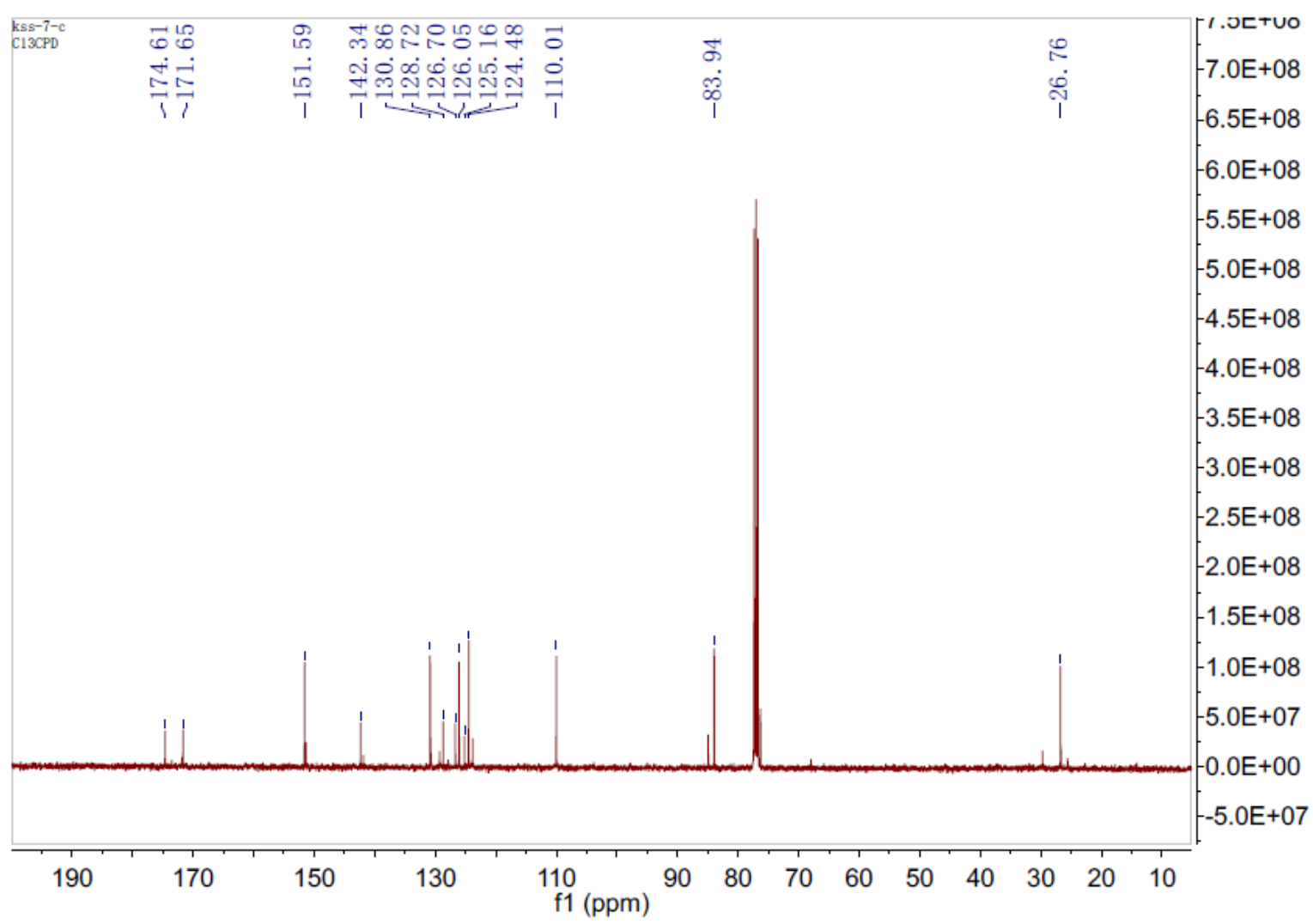

